\title{
Nutrient intake and patterns in the European Prospective Investigation into Cancer and Nutrition cohorts from 10 European countries
}

European Journal of Clinical Nutrition (2009) 63, S1-S2; doi:10.1038/ejcn.2009.122

This report is dedicated to two people, David Southgate and Sheila Bingham, who, between them both directly and indirectly have contributed hugely to the work reported here and to nutrition more widely. In terms of the Composition of Foods and Dietary Survey methodology they led the world. What was special about both of them was that they were such good people, always caring and supporting others, which enabled the sort of collaborative effort represented by this supplement on nutrient intakes and patterns in the European Prospective Investigation into Cancer and Nutrition (EPIC) cohorts. They will be sorely missed from the world of nutrition. I am sure that they would both appreciate the care and attention to detail exhibited in the work reported here. In fact, David Southgate helped in the writing of some of the papers.

The papers reported here represent different analyses undertaken by using a common data set. That data set comprised the 24-h dietary recalls completed by 3603435 - to 74-year-old men and women from 23 centres in 10 countries using the same standardized computerized interview programme (EPIC-Soft ${ }^{\mathbb{R}}$, International Agency for Research on Cancer, WHO, Lyon, France). These data were collected between 1995 and 2000 as part of the calibration of the EPIC cohort study. The key purpose of the collection of these data was to standardize or adjust for differences in techniques across study centres in the way in which the individual dietary data were collected at baseline, and to correct for measurement error and attenuate bias in relative risk estimates. It was, however, realized that these data were also valuable as a resource to characterize the behaviour of the European population based on a carefully standardized method. It may be that dietary patterns have changed in the last 10 years since the data were collected and the EPIC populations may not be representative of the national general populations; nevertheless, the results presented here will be a valuable resource and common benchmark for Europe. In EPIC, this information is particularly useful to understand and interpret better dietary exposure and its association with diseases (cancer and other endpoints), and to help formulate new hypotheses.
This supplement consists of 14 papers, with each paper written by up to 45 authors from 46 different institutions across Europe. The coordination of this activity was undertaken by Nadia Slimani from the Dietary Exposure Assessment Group, International Agency for Research on Cancer, Lyon, France. Her attention to detail ensured that there was a common protocol for the way in which data files were prepared, and subsequently how each writing group analysed and presented their results.

Each paper presents results that are broken down by age and gender, and region/study centre. Paper 1 describes the energy intake and sources of energy in the diet; papers 2-4 cover protein, carbohydrate and fat intakes. Paper 5 presents results for alcohol, paper 6 covers minerals, paper 7 covers water-soluble vitamins and paper 8 fat-soluble vitamins. There are three papers that explore the contribution that eating out (13), highly processed foods (11) and specific food group combinations (14) make to variation in nutrient intakes across the 10 EPIC studies. Paper 12 summarizes the use of dietary supplements and paper 10 presents data on glycaemic load and glycaemic index. There is one methodological paper (paper 9) that compares dietary intakes with urinary markers of nitrogen and potassium.

There was no north-south gradient in energy intake patterns; the average percentage of energy from fat was $34 \%$ and was the highest in Greece (40\%). In most centres cereals and cereal products were the largest contributors to energy intake. Total and animal protein intake was highest in Spanish men. Retinol intakes were highest in the northern countries, whereas vitamin $\mathrm{E}$ intakes were highest in southern countries. The level of $\beta$-carotene and vitamin $\mathrm{E}$ also showed some differences by level of education, smoking status and physical activity. There was a geographical gradient in iron intake, with higher intakes in southern than northern Europe and also around a twofold northsouth gradient in the contribution of fruits and vegetables to potassium intake.

Between countries, the mean percentage of dietary supplement use varied almost 10 -fold among women and even more among men. There was a clear north-south gradient in use, with higher consumption in the northern countries. 
From a set of 39 food groups, despite large variability in food and nutrient intake reported in EPIC, the variance of important nutrients is explained, to a large extent, by similar food group combinations across countries. The composition of the diet at home was different from that consumed out of home in southern countries, but was relatively similar in the north. When, however, the food grouping is divided more into level of processing, it was apparent that in Nordic and central European countries highly industrially processed foods dominated the diets and nutrient patterns, whereas in southern countries the contribution of these foods was more variable and associated with changing from traditional to more industrialized dietary patterns.

From a methodological perspective, there was variation in the reported level of underreporting mostly up to around $15 \%$, with one outlier at $32 \%$. This suggests the need for caution when interpreting the results either with or without underreporters included; there may be some potential for bias. For nitrogen and potassium, dietary compared with urinary estimates suggested that there was a reasonable agreement between the two at the group level, but not so good for nitrogen at the individual level.

It has been a privilege, albeit a sad one, to complete the task started by Sheila Bingham of guest editing with Nadia Slimani this supplement.

\section{Conflict of interest}

BM Margetts has received grant support from the Department of Health, UK, and Wellcome Trust.

BM Margetts, Guest Editor Professor of Public Health Nutrition, School of Medicine, University of Southampton, Southampton, UK E-mail: B.M.Margetts@soton.ac.uk 